\title{
Clinical features and prognosis of primary tracheal small cell carcinoma: a population-based analysis
}

\author{
Kun Chen ${ }^{1}$, Zhihao Yang ${ }^{1}$, Xinbo Zhang ${ }^{1}$, Tianhao Zhao ${ }^{1}$, Xiang Zhang ${ }^{1}$, Wenfeng Li ${ }^{2}$, Shanshan Yu ${ }^{2}$, \\ Deyao Xie ${ }^{1}$, Zhifeng $\mathrm{He}^{1}$, Dezhi Cheng ${ }^{1}$ \\ ${ }^{1}$ Department of Thoracic Surgery, The First Affiliated Hospital of Wenzhou Medical University, Wenzhou 325000, China; ${ }^{2}$ Department of \\ Chemoradiation Oncology, The First Affiliated Hospital of Wenzhou Medical University, Wenzhou 325000, China \\ Contributions: (I) Conception and design: All authors; (II) Administrative support: D Cheng; (III) Provision of study materials or patients: All authors; \\ (IV) Collection and assembly of data: K Chen; (V) Data analysis and interpretation: All authors; (VI) Manuscript writing: All authors; (VII) Final \\ approval of manuscript: All authors. \\ Correspondence to: Dezhi Cheng, MD. Department of Thoracic Surgery, The First Affiliated Hospital of Wenzhou Medical University, Southern \\ White Elephant Town, Ouhai District, Wenzhou 325000, China. Email: dezhicheng@sina.com.
}

Background: Primary tracheal small cell carcinoma (SCC) is an uncommon malignancy; therefore, its clinical features and prognosis are still unclear.

Methods: We used the population-based Surveillance, Epidemiology, and End Results (SEER) database to elucidate the clinical features and prognosis of primary tracheal SCC. The clinical features were assessed by using the chi-square test. Overall survival (OS) was computed using the Kaplan-Meier method, and a Cox proportional hazards analysis was performed to evaluate the prognostic factors.

Results: From 1973 to 2015, 1,392 primary tracheal tumor cases were reported in the SEER database, 75 (5.4\%) of which were SCC. Age, sex, race, extent of disease, lymph node involvement, surgery and radiation treatment were similar between patients with SCC and those with squamous cell carcinoma (SQC), but patients with SCC were more likely to receive chemotherapy $(65.8 \%$ vs. $28.2 \%$, respectively; $\mathrm{P}<0.001)$. The 1-, 3- and 5-year OS rates of patients with SCC were 37.8\%, 12.4\% and 7.1\%, respectively, and the median survival duration was 10.0 months, which was much worse than that observed for patients with other histopathological types of tracheal cancer. Among patients aged 60-100 years and those with regional lymph node involvement, the OS for patients with SQC was superior to that for patients with SCC (P=0.034 and $\mathrm{P}=0.016$, respectively). According to the multivariate analysis, age and lymph node involvement are independent prognostic factors of SCC.

Conclusion: Primary tracheal SCC is a rare carcinoma with a poor prognosis. Age and lymph node involvement are independent prognostic factors of SCC.

Keywords: Primary tracheal carcinoma; small cell carcinoma (SCC); prognosis; SEER database

Submitted Jul 30, 2019. Accepted for publication Nov 29, 2019.

doi: $10.21037 /$ tcr.2019.12.29

View this article at: http://dx.doi.org/10.21037/tcr.2019.12.29

\section{Introduction}

Primary tracheal malignant tumors are rare. The annual incidence of malignant tracheal tumors is approximately 0.142 in every 100,000 persons per year, accounting for $0.034 \%$ of all malignancies $(1,2)$. Squamous cell carcinoma (SQC) is the most common pathology (49\% of all primary tracheal carcinomas), followed by adenoid cystic carcinoma (ACC) (20\% of all primary tracheal carcinomas) (3).

Primary tracheal small cell carcinoma (SCC) is even more rare, and it is reported either as part of a large study or case reports (4-7). According to a nationwide survey, the incidence of SCC is $11.0 \%$ (1). Qiu et al. (7) reviewed the literature and found 90 cases of SCC among 
2,090 cases of primary tracheal cancer from 9 English language studies, showing that the incidence of SCC in the trachea is approximately $4.3 \%$. Primary tracheal SCC histologically falls within the spectrum of neuroendocrine tumors (8). Similar to small cell lung cancer (SCLC), immunohistochemistry can confirm a diagnosis of primary tracheal SCC with neuroendocrine markers, including neuron-specific enolase, synaptophysin, and chromogranin A $(9,10)$. The diagnosis of primary tracheal SCC should exclude the metastasis of pulmonary SCC via a workup (7). With the above method, a diagnosis can be readily made.

However, because of the rarity of primary SCC of the trachea, its clinical features and optimal management remain unclear, and a large database such as the Surveillance, Epidemiology, and End Results (SEER) database provides a unique opportunity to study this tumor. In this study, we used the SEER database to elucidate the clinical features and prognosis of primary tracheal SCC.

\section{Methods}

\section{Patient population}

We conducted this retrospective study using data retrieved from the SEER 18 registry database, which provides detailed cancer outcomes and represents $28 \%$ of the US population. We identified the primary tracheal tumors according to the primary site code C33.9. All the tumor cases studied in this article were from patients who were diagnosed with primary tracheal tumors between 1973 and 2015. The histology selection was limited to primary tracheal SCC, which was coded as $8041,8042,8043,8044$ and 8045 according to the International Classification of Disease for Oncology, third revision. In addition, the patients who were diagnosed with other types of primary tracheal cancer during the same period were also identified. Only patients with histologically confirmed disease were included in our study. We excluded patients whose survival time was unknown or whose diagnostic report was obtained from an autopsy or a death certificate. Ten of the 1,314 patients had no follow-up information $(0.7 \%)$ and were excluded from the survival analysis.

The variables included race, age, sex, year of diagnosis, primary tumor site, extent of tumor, lymph node involvement, histology, surgical data, survival status, survival months, vital status recode, chemotherapy and radiation.

\section{Definition of tumor data}

Because there is no American Joint Committee on Cancer (AJCC) tumor-node-metastasis (TNM) staging system for tracheal tumors and the tumor characteristics are defined by the EOD4, EOD10, and collaborative stage (CS) coding system in the SEER databases, all the patients were categorized by the following criteria: (I) extent of disease: localized (confined to the trachea) or regional (extension into adjacent tissue, including the arch of the aorta, azygos vein, right brachiocephalic vein, carotid sheath, common carotid artery, jugular arch, phrenic nerves, pretracheal fascia, recurrent laryngeal nerve, subclavian artery, and vagus nerve, or adjacent organs/structures, including the cricoid cartilage, esophagus, pleura, and not otherwise specified: parietal and visceral, right and left main bronchi, sternum, thymus, thyroid gland, and vertebral column, and further contiguous extension); and (II) lymph node involvement: no lymph nodes involved; regional lymph nodes involved (mediastinal, paratracheal and pretracheal); or distant lymph nodes involved.

\section{Statistical methods}

We employed the Student's test for continuous data. The chi-square test was used to compare the differences in categorical variables. The Kaplan-Meier method was used to generate survival curves. The differences between these curves were analyzed by the log-rank test. Overall survival (OS) was calculated from the date of diagnosis to the date of death or the last follow-up. Univariate and multivariate survival analyses were conducted using the Cox proportional hazards model. The corresponding hazard ratios (HRs) and $95 \%$ confidence intervals (CIs) were estimated from the model. Two-tailed $\mathrm{P}$ values $<0.05$ were considered statistically significant. Statistical analysis was performed using IBM SPSS 20.0 software (IBM Corporation, Chicago, IL, USA).

\section{Results}

\section{Patient characteristics}

From 1973 to 2015 , 1,392 primary tracheal tumor cases were registered in the SEER database, of which 73 were primary tracheal SCC. Primary tracheal SCC accounted for $5 \%$ of all cases of primary tracheal cancer. The cut-off date 
Table 1 Comparison of demographic and clinical characteristics of patients with small cell carcinoma and squamous cell carcinoma

\begin{tabular}{|c|c|c|c|}
\hline Variable & Small cell carcinoma & Squamous cell carcinoma & $P$ value \\
\hline Sex & & & 0.299 \\
\hline Male & $42(57.5)$ & $425(63.7)$ & \\
\hline Female & $31(42.5)$ & $242(36.3)$ & \\
\hline White & $62(84.9)$ & $559(83.8)$ & \\
\hline Black & $7(9.6)$ & $79(11.8)$ & \\
\hline Other & $4(5.5)$ & $29(4.3)$ & \\
\hline Extent & & & 0.435 \\
\hline Distant disease & $14(19.2)$ & $94(14.1)$ & \\
\hline Unknown & $9(12.3)$ & $117(17.5)$ & \\
\hline Lymph node involvement & & & 0.894 \\
\hline No lymph nodes involved & $28(38.4)$ & $278(41.7)$ & \\
\hline Regional lymph nodes involved & $17(23.3)$ & $133(19.9)$ & \\
\hline Distant lymph nodes involved & $4(5.5)$ & $32(4.8)$ & \\
\hline Unknown & $24(32.9)$ & $224(33.6)$ & \\
\hline Radiation use & & & 0.278 \\
\hline Yes & $15(20.5)$ & $217(32.5)$ & \\
\hline Unknown & $19(26.0)$ & $127(19.0)$ & \\
\hline Chemotherapy & & & $<0.001$ \\
\hline No/unknown & $25(34.2)$ & $479(71.8)$ & \\
\hline Yes & $48(65.8)$ & $188(28.2)$ & \\
\hline
\end{tabular}

of the follow-up was December 31, 2015.

We identified 73 patients with primary SCC and 1,241 patients with other types of primary tracheal cancer (including 668 patients with SQC and 202 patients with ACC) diagnosed between 1973 and 2015 in the SEER 18 registry database. As summarized in Table 1, the mean age was 63 years for patients with SCC and 66 years for patients with SQC $(\mathrm{P}=0.106)$. Among the SCC patients,
$58 \%$ were females, and $42 \%$ were males. There was no significant difference between SQC and SCC patients with regard to sex $(\mathrm{P}=0.299)$. There were also no significant differences between SCC and SQC patients with regard to race $(\mathrm{P}=0.784)$, extent of disease $(\mathrm{P}=0.435)$, lymph node involvement $(\mathrm{P}=0.894)$, surgery $(\mathrm{P}=0.083)$ or radiation treatment $(\mathrm{P}=0.278)$. However, patients with SCC were more likely to receive chemotherapy $(65.8 \%$ vs. $28.2 \%$, 

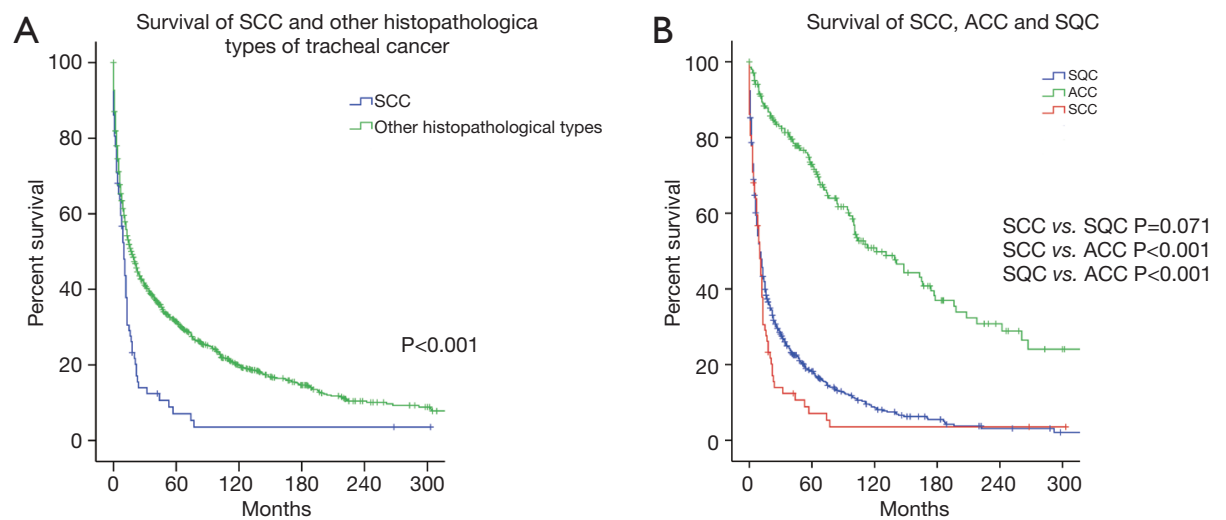

Figure 1 Kaplan-Meier curves for OS of SCC, ACC and SQC. (A) The OS of patients with SCC and other histopathological types of tracheal cancer; (B) the OS of SCC, ACC, and SQC patients. SCC, small cell carcinoma; SQC, squamous cell carcinoma; OS, overall survival; ACC, adenoid cystic carcinoma.
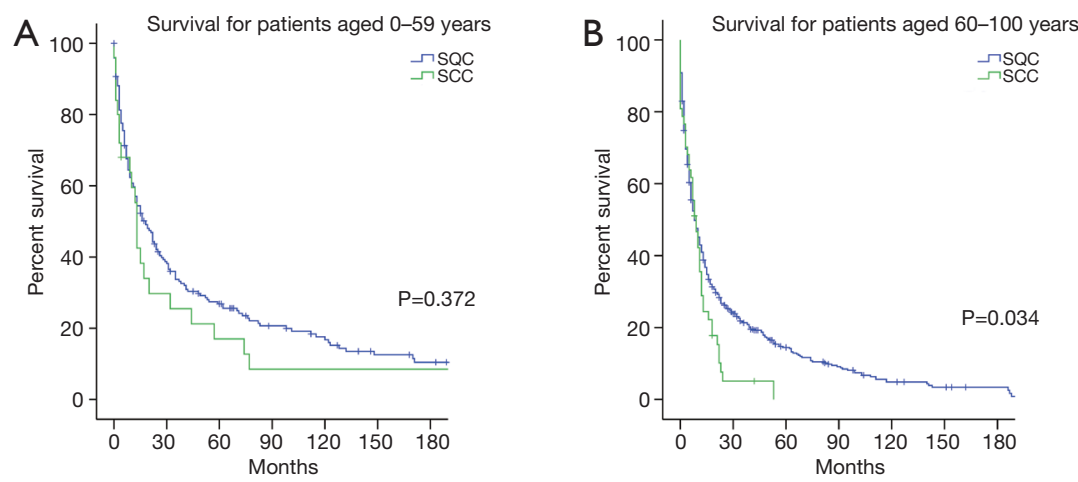

Figure 2 Kaplan-Meier curves of OS for patients with SCC and SQC aged 0-59 years (A) or 60-100 years (B). SCC, small cell carcinoma; SQC, squamous cell carcinoma; OS, overall survival.

respectively; $\mathrm{P}<0.001)$ than $\mathrm{SQC}$ patients.

\section{Clinical outcomes differ between SCC and other histopathological types of tracheal cancer}

The 1-, 3- and 5-year OS rates of patients with SCC were $37.8 \%, 12.4 \%$ and $7.1 \%$, respectively, whereas those for patients with other histopathological types of tracheal cancer were $55.8 \%, 38.7 \%$ and $31.6 \%$, respectively. The median survival duration of all SCC patients was 10.0 (95\% CI: 7.3-12.7) months, which was much worse than that of patients with other histopathological types of tracheal cancer [median survival: 18.0 (95\% CI: 15.2-20.8) months]. In the comparison between SCC and other histopathological types of tracheal cancer, it was obvious that SCC had a worse prognosis (Figure $1 A$ ). We also aimed to determine the difference in OS among SCC,
ACC and SQC patients. In the current study, the median survival times were 10.0 (95\% CI: 7.3-12.7), 122.0 (95\% CI: $90.5-153.5$ ) and 10.0 (95\% CI: 8.3-11.7) months for SQC, ACC and SCC patients, respectively. The results showed that patients with ACC had a better OS than those with SCC and SQC (SCC vs. ACC P<0.001; SQC vs. ACC $\mathrm{P}<0.001)$. However, no significant difference was identified between SCC and SQC patients $(\mathrm{P}=0.071)$ (Figure $1 B)$. We then analyzed the difference in clinical outcomes between SCC and SQC of the trachea in subgroups stratified by age and lymph node involvement. Among patients aged 60-100 years at presentation, the OS for patients with SQC was superior to that for patients with SCC $(\mathrm{P}=0.034)$, whereas the prognosis of those aged $0-59$ years was not affected by the histopathological type ( $\mathrm{P}=0.372$; Figure 2). In the regional lymph node involvement subgroup, the median survival of patients with SQC was 11.9 (95\% CI: 

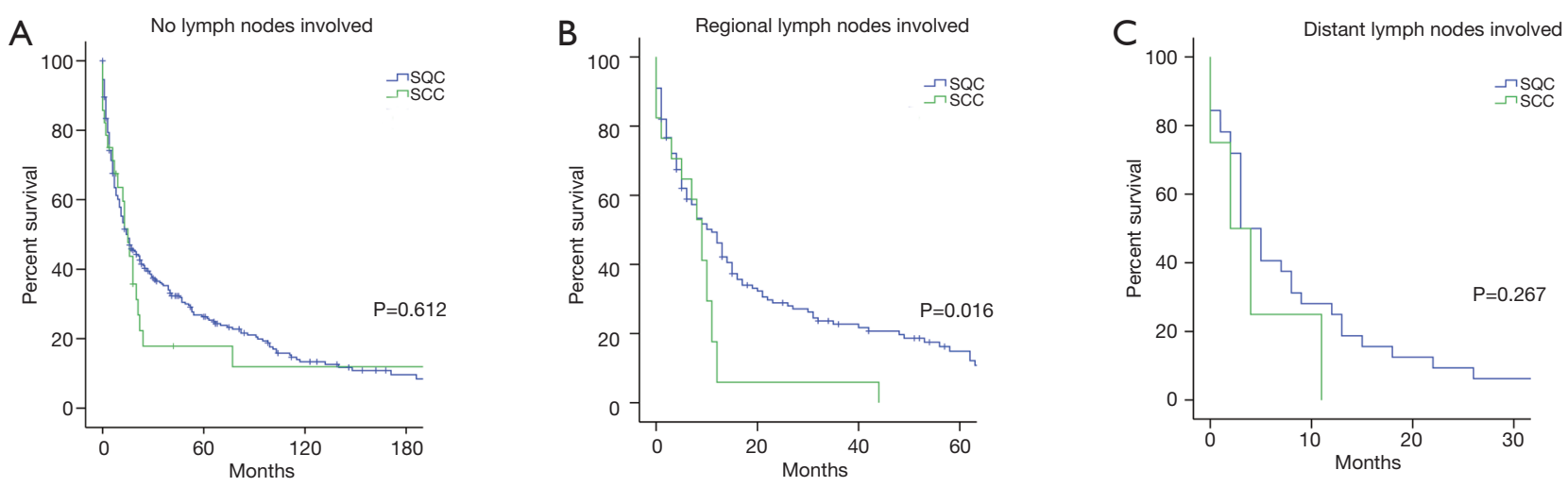

Figure 3 Kaplan-Meier curves of OS for patients with primary tracheal carcinoma and no lymph node involvement (A), regional lymph node involvement (B) or distant lymph node involvement (C). SCC, small cell carcinoma; SQC, squamous cell carcinoma; OS, overall survival.

7.6-14.4) months, which was superior to the 9.0 (95\% CI: 6.3-11.7) months for patients with SCC ( $\mathrm{P}=0.016$; Figure 3). The median survival of SQC patients with no lymph node involvement was 14.0 (95\% CI: 9.1-18.9) months, and that for SCC patients with no lymph node involvement was 15.0 (95\% CI: $10.2-19.8)$ months $(\mathrm{P}=0.612)$. The median survival for SQC patients with distant lymph node involvement was 3.0 (95\% CI: 1.3-4.7) months, and that for SCC patients with distant lymph node involvement was 2.0 (95\% CI: 0-5.9) months ( $\mathrm{P}=0.267)$.

\section{Prognostic factors of SCC}

Univariate and multivariate Cox proportional hazard models were used to identify the prognostic factors associated with OS (Table 2). In the univariate analysis, the race and sex of the patients were not significantly associated with poor survival ( $\mathrm{P}=0.056$ and 0.399 , respectively). Additionally, we noticed that radiation and surgery were not prognostic factors in tracheal small cell tumor patients. However, age, extent of disease, lymph node involvement and chemotherapy treatment were significantly associated with survival. Older patients had worse survival outcomes than younger patients ( $\mathrm{HR}=1.025,95 \% \mathrm{CI}: 1.007-1.043$, $\mathrm{P}=0.004)$. Patients with distant disease had worse survival outcomes than patients with localized disease $(\mathrm{HR}=$ 1.991, 95\% CI: 1.016-3.902, P=0.045). Patients without pathologically involved lymph nodes showed a trend towards survival benefits compared with patients with regional lymph nodes ( $\mathrm{HR}=2.509,95 \% \mathrm{CI}$ : 1.285-4.898; $\mathrm{P}=0.007)$ or distant lymph nodes $(\mathrm{HR}=4.247$, 95\% CI:
1.399-12.895; $\mathrm{P}=0.011)$. Additionally, chemotherapy treatments significantly improved survival ( $\mathrm{HR}=0.563,95 \%$ CI: $0.339-0.935, \mathrm{P}=0.026$ ). In our subsequent multivariate analysis after accounting for age, extent of disease, lymph node involvement and chemotherapy, we found that age and lymph node involvement were independent prognostic factors (Table 2).

\section{Discussion}

We conducted a population-based retrospective study to reveal the clinicopathological characteristics and survival outcomes of primary tracheal SCC. In our study, the incidence of primary SCC of the trachea was approximately $5 \%$, which is similar to the results of a study by Qiu et al. (7). We found that the mean age of the patients with primary tracheal SCC was 63, which is somewhat older than the age reported in the literature (61.3 years) (11); the patients with primary tracheal SCC was younger than the patients with SQC, although there was no significant difference. Among those with SCC, 58\% were female, and $42 \%$ were male. In our study, the male-to-female ratio was 1.35:1 for SCC and 1.76:1 for SQC, and there were no significant differences in sex between SQC and SCC. This finding may support the hypothesis that SQC and SCC are associated with smoking. In this study, Caucasian patients accounted for the largest proportion of SCC patients (84.9\%), which is consistent with the distribution of races in the Western population and is also not significantly different from the race distribution of SQC. Surprisingly, there was no significant difference between SCC and SQC patients in 
Table 2 Univariate and multivariate analyses of overall survival of tracheal small cell carcinoma

\begin{tabular}{|c|c|c|c|c|c|}
\hline Covariate & $\mathrm{N}$ & \multicolumn{2}{|l|}{ Univariate } & \multicolumn{2}{|c|}{ Multivariate } \\
\hline Age & 72 & $1.025(1.007-1.043)$ & 0.004 & $1.025(1.006-1.044)$ & 0.008 \\
\hline \multicolumn{6}{|l|}{ Sex } \\
\hline Male & 41 & 1 & - & - & - \\
\hline \multicolumn{6}{|l|}{ Race } \\
\hline White & 61 & 1 & - & - & - \\
\hline Black & 7 & $2.137(0.948-4.818)$ & 0.067 & - & - \\
\hline Other & 4 & $0.415(0.126-1.368)$ & 0.148 & - & - \\
\hline \multicolumn{6}{|l|}{ Extent } \\
\hline Regional disease & 21 & $1.054(0.582-1.907)$ & 0.863 & $0.846(0.446-1.604)$ & 0.609 \\
\hline Distant disease & 14 & $1.991(1.016-3.902)$ & 0.045 & $1.774(0.805-3.910)$ & 0.155 \\
\hline Unknown & 9 & $1.532(0.675-3.476)$ & 0.308 & $1.759(0.759-4.077)$ & 0.188 \\
\hline \multicolumn{6}{|l|}{ Lymph node involvement } \\
\hline No lymph nodes involved & 28 & 1 & - & 1 & - \\
\hline Regional lymph nodes involved & 17 & $2.509(1.285-4.898)$ & 0.007 & $2.456(1.251-4.823)$ & 0.009 \\
\hline Distant lymph nodes involved & 4 & $4.247(1.399-12.895)$ & 0.011 & $4.059(1.341-12.290)$ & 0.013 \\
\hline Unknown & 23 & $1.475(0.814-2.672)$ & 0.2 & $1.421(0.783-2.577)$ & 0.248 \\
\hline Yes & 15 & $0.54(0.278-1.05)$ & 0.069 & - & - \\
\hline Unknown & 19 & $1.119(0.638-1.963)$ & 0.685 & - & - \\
\hline \multicolumn{6}{|l|}{ Chemotherapy } \\
\hline No/unknown & 25 & 1 & - & 1 & - \\
\hline Yes & 47 & $0.563(0.339-0.935)$ & 0.026 & $0.665(0.355-1.247)$ & 0.204 \\
\hline
\end{tabular}

$\mathrm{HR}$, hazard ratios; $\mathrm{Cl}$, confidence interval.

terms of the distributions of the extent of disease and lymph node involvement, and only $19.2 \%$ SCC patients had distant disease, which was lower than the corresponding percentage for patients with SCLC (12). One possible reason is that extrapulmonary small cell carcinoma (EPSCC) is a clinical pathologic entity distinct from small cell lung carcinoma (13). In addition, hemoptysis is the main symptom of malignant tracheal tumors (14) and may lead to an earlier diagnosis. In terms of treatment, patients with SCC were more likely to receive chemotherapy than SQC patients. SCC patients underwent fewer surgical treatments, although the difference was not statistically significant, 
which may be due to the treatment of SCLC.

In the current study, the 1-, 3-, and 5-year OS rates for patients with SCC were $37.8 \%, 12.4 \%$ and $7.1 \%$, respectively, compared to $55.8 \%, 38.7 \%$ and $31.6 \%$ for patients with other histopathological types of tracheal cancer; the median survival of all SCC patients was 10.0 months, whereas the median survival of patients with other histopathological types of tracheal cancer was 18.0 months. The prognosis of SCC patients was worse than that of patients with other histopathological types of tracheal cancer. Then, we compared the survival outcomes of different histopathological types of tracheal tumors. We found that ACC had expectedly better OS than SCC and SQC, but surprisingly, no significant difference was identified between SCC and SQC. These two types of tracheal carcinoma may have the same poor outcome. Licht et al. (15), whose study involved 8 patients with SCC, reported that the 1-year OS survival rate of patients with SCC was $0 \%$, which was lower than our results. Gelder et al. (11), whose study involved 16 patients with SCC, reported that the 1- and 5-year OS rates for patients with SCC were $32.4 \%$ and $0 \%$, respectively, which were lower than those obtained in the present study. This inconsistency may result from the small sample size of the previous study. To further study the clinical outcome differences between SCC and SQC, we evaluated subgroups with univariate analyses. We found that for patients aged 60-100 years, the OS of those with SQC was superior to that of patients with SCC, whereas the prognosis of those aged 0-59 years was not affected by the histopathological type. In the regional lymph node involvement subgroup, the median survival of patients with SQC was superior to that of patients with SCC. However, the prognoses of those with no lymph node involvement and distant lymph node involvement were not affected by the histopathological type. Although the overall difference in OS between SCC and SQC did not reach statistical significance, which is partly because of the small sample size, we found that the OS for patients with SQC was superior to that for patients with SCC in the older and regional lymph node involvement subgroup.

In the univariate and multivariate analyses, the results suggest that older age and lymph node involvement were independent factors for the prognosis of SCC. Bhattacharyya et al. (16) suggested that positive nodal disease had a significant negative effect on OS for primary tracheal malignancies, as positive regional nodal disease diminished survival by greater than $50 \%$ compared to N0 disease. Wen et al. (17) also indicated that lymph node involvement was important for the prognosis of primary tracheal tumors. Our study confirms this view in primary tracheal small cell tumors.

However, sex, race, radiation and surgery were not correlated with the OS of SCC. The optimal treatment for SCC is still unclear. The role of surgical resection in the treatment of SCLC remains controversial, and the National Comprehensive Cancer Network (NCCN) guidelines support that surgical resection is only appropriate for select patients with early-stage SCLC (18). Qiu et al. (7) suggested that surgical resection should be performed and followed by postoperative adjuvant therapy in cases of cervical tracheal SCC. Wen et al. (17) suggested that patients with primary tracheal carcinoma who underwent surgery had better survival outcomes than those who did not undergo surgery. Our results revealed that surgical resection had a tendency to achieve longer survival in patients, although this trend was not statistically significant, which may partly be explained by the limited sample size of our study. In addition, surgical resection for the treatment of SCC may include palliative or non-radical surgery. In the current study, radiotherapy had a tendency to achieve longer survival in patients, although this trend was also not statistically significant. In our cohort, the effect of chemotherapy was statistically significant in the univariate analysis but not in the multivariate analysis. However, according to previously published studies, radiochemotherapy is often used to treat SCC. Luo et al. (9) reported a case of primary tracheal SCC coexisting with esophageal cancer, which was treated with sequential chemoradiation involving four cycles of systemic chemotherapy with an etoposide and carboplatin (EC) regimen followed by radiotherapy with $60 \mathrm{~Gy}$, and showed a complete response of the tracheal tumor and right supraclavicular lymph node. Hagiwara et al. (19) also reported that a patient was treated with chemotherapy followed by radiation therapy, and the therapeutic outcome was deemed a complete response. Furthermore, our findings indicate that chemotherapy, surgery and radiotherapy did not significantly benefit OS, but this study was unable to exclude the possibility that these treatments were effective in prolonging progression-free survival (PFS). Regrettably, the SEER database did not provide any recurrence data. Our study suggests that patients with distant disease had worse survival outcomes than patients with localized disease in the univariate analysis, although the extent of disease was not an independent prognostic factor in the multivariate analysis.

This study had several limitations. First, 73 cases of 
tracheal SCC were identified in the SEER database. Therefore, the results of some analyses may not be significant because of the small sample size. These results need to be confirmed by future studies with a larger sample size. Second, some important variables, such as smoking history, laboratory test results, tumor recurrence, imaging findings and the detailed chemotherapy regimens, were not available in the SEER database, which may affect the survival analysis. Third, the patient database of our study was retrospectively collected, which leads to inherent selection bias. Hence, caution should be applied when interpreting our results.

\section{Conclusions}

Primary tracheal SCC is a relatively rare carcinoma with a poor prognosis. The clinical characteristics of SCC are similar to those of SQC. Age and lymph node involvement were independent prognostic factors of SCC. To understand primary tracheal SCC more thoroughly, more cases are required.

\section{Acknowledgments}

The authors are grateful to all the staff at the National Cancer Institute (USA) for their contribution to the SEER program.

Funding: The study was supported by the Natural Science Foundation of Zhejiang Province (No. LY14H160042) and Wenzhou Public Welfare Science and Technology Plan (No. Y20180175).

\section{Footnote}

Conflicts of Interest: The authors have completed the ICMJE uniform disclosure form (available at http://dx.doi. org/10.21037/tcr.2019.12.29). The authors have no conflicts of interest to declare.

Ethical Statement: The authors are accountable for all aspects of the work in ensuring that questions related to the accuracy or integrity of any part of the work are appropriately investigated and resolved.

Open Access Statement: This is an Open Access article distributed in accordance with the Creative Commons Attribution-NonCommercial-NoDerivs 4.0 International License (CC BY-NC-ND 4.0), which permits the non- commercial replication and distribution of the article with the strict proviso that no changes or edits are made and the original work is properly cited (including links to both the formal publication through the relevant DOI and the license). See: https://creativecommons.org/licenses/by-nc-nd/4.0/.

\section{References}

1. Honings J, van Dijck JA, Verhagen AF, et al. Incidence and treatment of tracheal cancer: a nationwide study in the Netherlands. Ann Surg Oncol 2007;14:968-76.

2. Madariaga MLL, Gaissert HA. Overview of malignant tracheal tumors. Ann Cardiothorac Surg 2018;7:244-54.

3. Gao H, He X, Du J, et al. Competing risk analysis of primary tracheal carcinoma based on SEER database. Cancer Manag Res 2019;11:1059-65.

4. Wang $\mathrm{H}$, Zhang $\mathrm{N}$, Li D, et al. Interventional Bronchoscopies for the Treatment of 112 Cases with Complex Malignant Central Airway Diseases Involved in Bifurcation. Zhongguo Fei Ai Za Zhi 2016;19:854-8.

5. Mallick S, Benson R, Giridhar P, et al. Demography, patterns of care and survival outcomes in patients with malignant tumors of trachea: A systematic review and individual patient data analysis of 733 patients. Lung Cancer 2019;132:87-93.

6. Yusuf M, Gaskins J, Trawick E, et al. Effects of adjuvant radiation therapy on survival for patients with resected primary tracheal carcinoma: an analysis of the National Cancer Database. Jpn J Clin Oncol 2019;49:628-38.

7. Qiu J, Lin W, Zhou ML, et al. Primary small cell cancer of cervical trachea: a case report and literature review. Int J Clin Exp Pathol 2015;8:7488-93.

8. Moores D, Mane P. Pathology of Primary Tracheobronchial Malignancies Other than Adenoid Cystic Carcinomas. Thorac Surg Clin 2018;28:149-54.

9. Luo W, Luo G, Gong Y, et al. Synchronous primary cancers of trachea and esophagus and ventricular tachycardia. Chin J Cancer Res 2014;26:345-50.

10. Yang H, Liu L, Zhou C, et al. The clinicopathologic of pulmonary adenocarcinoma transformation to small cell lung cancer. Medicine (Baltimore) 2019;98:e14893.

11. Gelder CM, Hetzel MR. Primary tracheal tumours: a national survey. Thorax 1993;48:688-92.

12. Dores GM, Qubaiah O, Mody A, et al. A populationbased study of incidence and patient survival of small cell carcinoma in the United States, 1992-2010. BMC Cancer 2015;15:185.

13. Yasumatsu R, Nakashima T, Yamauchi M, et al. 
Extrapulmonary small cell carcinoma in head and neck. J

Laryngol Otol 2015;129 Suppl 2:S83-85.

14. Koul R, Alomrann R, Rathod S, et al. Clinical Characteristics and Prognosis of Primary Tracheal Cancer: A Single Institution Experience. Int J Hematol Oncol Stem Cell Res 2018;12:298-302.

15. Licht PB, Friis S, Pettersson G. Tracheal cancer in Denmark: a nationwide study. Eur J Cardiothorac Surg 2001;19:339-45.

16. Bhattacharyya N. Contemporary staging and prognosis for primary tracheal malignancies: a population-based analysis.
Otolaryngol Head Neck Surg 2004;131:639-42.

17. Wen J, Liu D, Xu X, et al. Nomograms for predicting survival outcomes in patients with primary tracheal tumors: a large population-based analysis. Cancer Manag Res 2018;10:6843-56.

18. Kalemkerian GP, Loo BW, Akerley W, et al. NCCN Guidelines Insights: Small Cell Lung Cancer, Version 2.2018. J Natl Compr Canc Netw 2018;16:1171-82.

19. Hagiwara E, Gon Y, Hayashi K, et al. Evaluation of airway resistance in primary small cell carcinoma of the trachea by MostGraph: a case study. J Thorac Dis 2016;8:E702-6.

Cite this article as: Chen $\mathrm{K}$, Yang $Z$, Zhang $\mathrm{X}$, Zhao $\mathrm{T}$, Zhang X, Li W, Yu S, Xie D, He Z, Cheng D. Clinical features and prognosis of primary tracheal small cell carcinoma: a population-based analysis. Transl Cancer Res 2020;9(2):882890. doi: $10.21037 /$ tcr.2019.12.29 